\title{
In situ Strain Mapping of Planar Slip in 304 Stainless Steel
}

Thomas C. Pekin ${ }^{1,2}$, Christoph Gammer ${ }^{3}$, Jim Ciston ${ }^{2}$, Colin Ophus ${ }^{2}$ and Andrew M. Minor ${ }^{1,2}$

${ }^{1 .}$ Department of Materials Science \& Engineering, University of California, Berkeley, CA 94720, USA.

2. National Center for Electron Microscopy, Molecular Foundry, Lawrence Berkeley National Laboratory, Berkeley, CA 94720, USA.

3. Erich Schmid Institute of Materials Science, Austrian Academy of Sciences, Jahnstrasse 12, 8700 Leoben, Austria.

The direct observation of dislocations by electron microscopy, both in and ex situ, has provided invaluable information that has been used to develop our understandings of the nanoscale origins of deformation [1,2]. In metallic materials, planar slip is a common deformation mechanism and has a direct consequence on the material's macroscale deformation behavior [3,4]. However, the causes of localized or 'planar' slip are not well understood. Of the many theories that exist [5,6], a prevailing one relies on the presence of short range order (SRO) as an inhibitor of more three-dimensional or 'wavy' slip [4,7]. As a dislocation moves through the matrix, the destruction of the short range order provides a softening of that specific slip plane, allowing for additional dislocations to propagate along the same slip plane. In some systems, it has been shown that this planar slip can cause antiphase boundaries resulting in a lattice relaxation, and therefore strain contrast in conventional transmission electron microscopy (TEM)[8]. However, direct quantification of this strain has not been performed.

Using scanning nanobeam election diffraction (NBED) and a fast detector, a time series of strain maps during an in situ deformation experiment of an austenitic (304) stainless steel was acquired [9]. After the analysis of the resulting $\sim 100,000$ diffraction patterns [10], a positive lattice dilation in the region behind the first dislocation involved with planar slip was observed to follow the dislocation as it moved across the field of view (Figure 1), which could correlate to the destruction of short range order. Compared to previous experiments [8], this type of experiment provides much richer information in the form of time-resolved quantifiable strain maps, allowing for the observation and analysis of dynamic processes. Figure 2 shows a transmission electron micrograph of the same region after testing, confirming the existence of planar slip, as well as showing the ability to perform traditional TEM techniques using the exact same sample after in situ strain mapping. The quantification of the strain field resulting from planar slip will be discussed in light of the potential implications for basic mechanisms of dislocation plasticity in metals.

\section{References:}

[1] M. Whelan et al, Proceedings of the Royal Society A: Mathematical, Physical, and Engineering Sciences 240 (1957), p. 524-38.

[2] Z. W. Shan et al, Nature Materials 7 (2007), p. 115-9.

[3] J. Nilsson, Scripta Metallurgica 17 (1983), p. 593-6.

[4] V. Gerold and H. Karnthaler, Acta Metallurgica 37 (1989), p. 2177-83.

[5] S. Hong and C. Laird, Acta Metallurgica et Materiala 38 (1990), p. 1581-94.

[6] Z. Wang, Philosophical Magazine 84 (2004), p. 351-79.

[7] N. Clemént, D. Caillard, and J. Martin, Acta Metallurgica 32 (1984), p. 961-75.

[8] G. van Tendeloo and S. Amelinckx, Physica Status Solidi (a) 27 (1975), p. 93-8. 
[9] C. Gammer et al, Applied Physics Letters 109 (2016), p. 081906.

[10] T. C. Pekin et al, Ultramicroscopy (2017) in press.

[11] The authors acknowledge support by the Director, Office of Science, Office of Basic Energy Sciences, Materials Sciences and Engineering Division, of the U.S. Department of Energy under Contract No. DEAC02-05-CH11231 under the Mechanical Behavior of Materials program. CG acknowledges support by the Austrian Science Fund (FWF):[J3397], Work at the Molecular Foundry was supported by the Office of Science, Office of Basic Energy Sciences, of the U.S. Department of Energy under Contract No. DE-AC02$05 \mathrm{CH} 11231$.
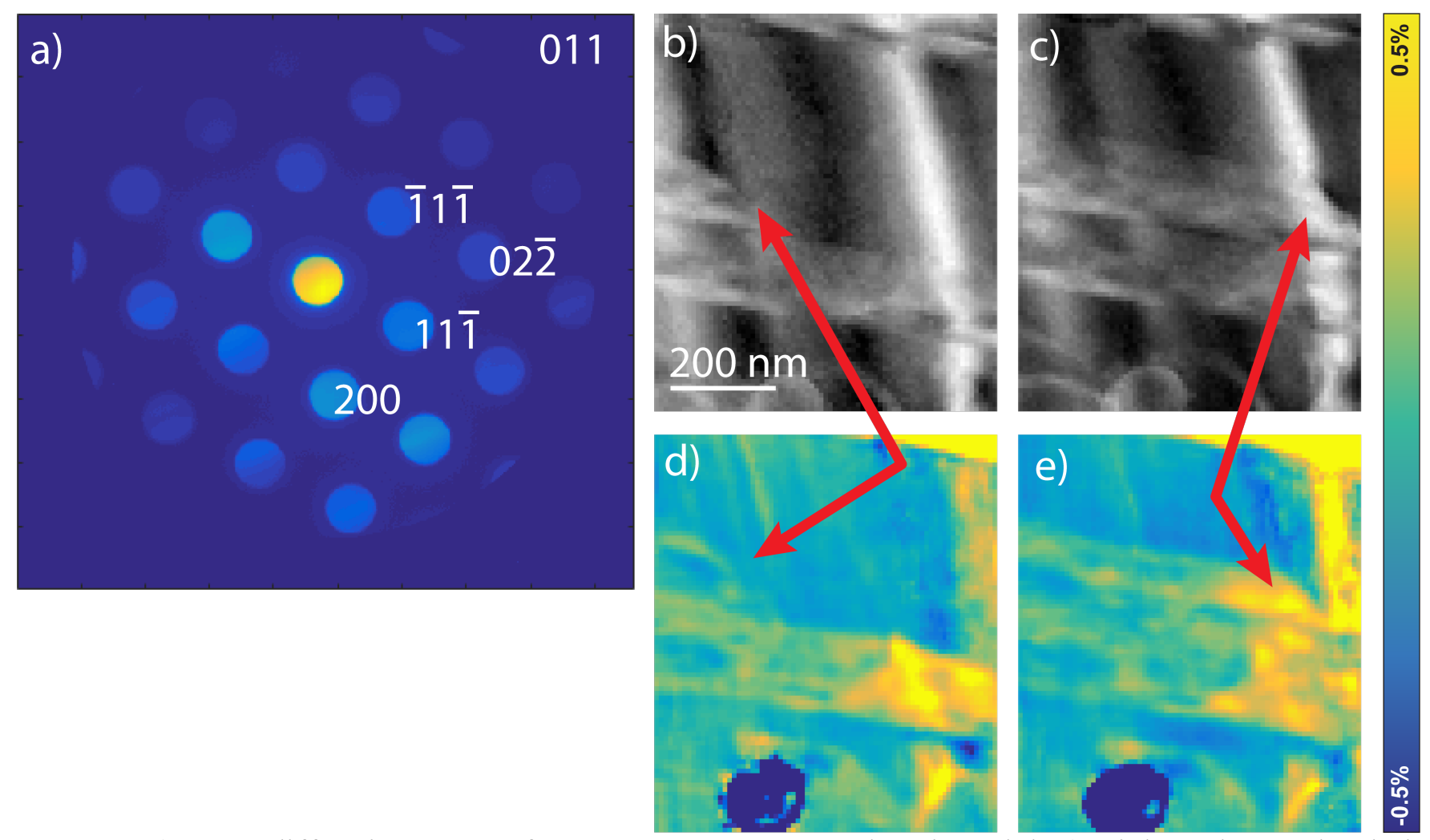

Figure 1 a) Mean diffraction pattern from NBED scan, correctly oriented. b-c) High angle annular dark field images at two different frames of the in situ straining video. Notice the top dislocation move from left to right as indicated by the red arrows in all frames. d-e) Lattice dilation $\left(\left(\varepsilon_{200}+\varepsilon_{02-2}\right) / 2\right)$ obtained from strain mapping corresponding to b) and c). Notice that behind the leading dislocation, a small dilation exists. This is also present in the slip plane below the moving dislocation, which was not observed to move.

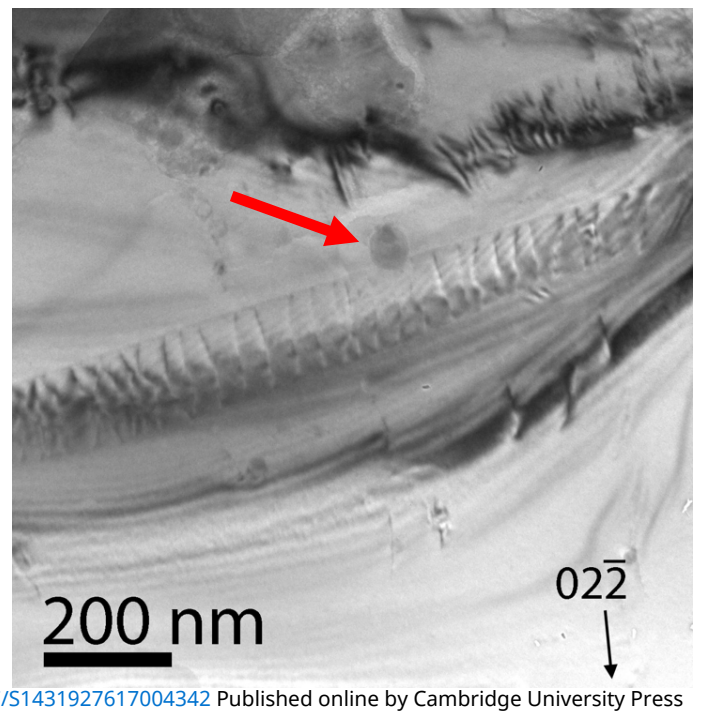

Figure 2 Brightfield TEM image of planar slip region. The dislocations are clearly planar in nature. Notice the same precipitate (red arrow) as seen in the HAADF and strain mapping images (and in the strain maps as a blue circle). 